\title{
HOLOMORPHIC CURVES IN LORENTZIAN CR-MANIFOLDS
}

BY

\author{
ROBERT L. BRYANT ${ }^{1}$
}

\begin{abstract}
A CR-manifold is said to be Lorentzian if its Levi form has one negative eigenvalue and the rest positive. In this case, it is possible that the CR-manifold contains holomorphic curves. In this paper, necessary and sufficient conditions are derived (in terms of the "derivatives" of the CR-structure) in order that holomorphic curves exist. A "flatness" theorem is proven characterizing the real Lorentzian hyperquadric $Q_{5} \subseteq \mathbf{C} P^{3}$, and examples are given showing that nonflat Lorentzian hyperquadrics can have a richer family of holomorphic curves than the flat ones.
\end{abstract}

0. Introduction. In the study of CR-manifolds, the presence of holomorphic submanifolds has a fundamental effect on the behavior of solutions of the natural PDE associated to a given CR-manifold. In fact, the holomorphic submanifolds play a role analogous to Monge characteristics in the theory of second order partial differential equations for one function of $n$-variables. The elliptic theory, i.e., no Monge characteristics, corresponds under this analogy to the strictly pseudo-convex CR-manifolds, where holomorphic submanifolds are forbidden by the positive definite behavior of the Levi form. The classical wave equation corresponds to the Lorentzian CR-manifolds (see $\S 1$ below) where the maximal isotropic subspaces of the holomorphic tangent space are complex lines. Thus, two dimensional complex submanifolds are forbidden by the signature of the Levi form, but the possibility of holomorphic curves remains. In this paper, we solve the problem of determining when holomorphic curves exist in a Lorentzian CR-manifold. Moreover, we derive an upper bound on the dimension of the parameter space of the holomorphic curves in a Lorentzian CR-manifold and give an example to show that this upper bound can be achieved.

The fundamental tools to be used are the notions of differential systems and prolongation. We will not need to assume any previous knowledge of these subjects, though the reader may wish to acquaint himself with the motivating ideas by consulting [Bryant-Chern-Griffiths].

The author would like to express his gratitude to Eric Bedford for acquainting him with the problem and supplying him with valuable references and to James Faran and Nancy Stanton for several illuminating discussions on CR-manifolds.

Received by the editors February 19, 1981.

1980 Mathematics Subject Classification. Primary 32F25; Secondary 53C10.

Key words and phrases. Prolongation, CR-structure, Levi form, holomorphic curve, Lorentzian hyperquadric.

${ }^{1}$ Research supported by grant MCS\# 80-03237. 
1. CR-manifolds. The basic model of a CR-manifold is a real hypersurface $M^{2 n+1}$ in $\mathbf{C}^{n+1}$. We may assume that $M=\rho^{-1}(0)$ where $\rho: \mathbf{C}^{n+1} \rightarrow \mathbf{R}$ takes on $0 \in \mathbf{R}$ as a regular value, i.e., $d \rho_{\left.\right|_{x}} \neq 0$ for all $x \in M$.

We let $A_{p q}\left(\mathbf{C}^{n+1}\right)$ be the complex-valued $C^{\infty}$-forms on $\mathbf{C}^{n+1}$ of type $(p, q)$ (see [Chern]). If $z^{0}, \ldots, z^{n}$ are holomorphic coordinates on $\mathbf{C}^{n+1}$, then $A_{10}\left(\mathbf{C}^{n+1}\right)$ is spanned, as a $C^{\infty}\left(\mathbf{C}^{n+1}\right)$-module, by the forms $d z^{\alpha}(\alpha=0, \ldots, n)$. We define

$$
I(M)=\left\{\omega_{\left.\right|_{M}} \mid \omega \in A_{10}\left(\mathbf{C}^{n+1}\right)\right\} \subseteq \Omega_{\mathbf{C}}^{1}(M),
$$

where $\Omega_{C}^{1}(M)$ is the set of (smooth) complex-valued 1-forms on $M$. When there is no danger of confusion, we simply write $I$ instead of $I(M)$. We note that $I \subseteq \Omega_{\mathrm{C}}^{1}(M)$ is a complex Pfaffian system of (complex) rank $n+1$. The important properties of $I$ are

(a) $I$ is differentially closed, i.e. $d \omega \equiv 0 \bmod I$ for all $\omega$ in $I$,

(b) $I+\bar{I}=\Omega_{\mathrm{C}}^{1}(M)$,

(c) $I \cap \bar{I}$ is of (complex) rank 1 .

Now we may describe $I \cap \bar{I}$ as follows: since $\rho \equiv 0$ on $M$, we have $d \rho \equiv 0$. Therefore $d \rho=\partial \rho+\bar{\partial} \rho=0$ on $M$. The assumption that 0 is a regular value of $\rho$ implies that $\partial \rho$ never vanishes on $M$. It follows that $\partial \rho$ generates $I \cap \bar{I}$ over $C_{\mathbf{C}}^{\infty}(M)$.

With the above as motivating example, we make the definition

Definition 1.1. A (smooth) CR-manifold is a pair $(M, I)$ where $M$ is a smooth manifold of dimension $2 n+1$ and $I \subseteq \Omega_{C}^{1}(M)$ is a Pfaffian system which satisfies

(a) $I$ has complex rank $n+1$ at every point of $M$,

(b) $d \omega \equiv 0 \bmod I$ for all $\omega \in I$,

(c) $I \cap \bar{I}$ has complex rank 1 at every point of $M$.

REMARKS. To be completely honest, we should call the above pair a maximally complex CR-manifold (of dimension $2 n+1$ ). Since no other kind of CR-manifold will occur in this paper, we omit the qualifying adjective phrase "maximally complex".

In the analytic category, it is known, cf. [Chern-Moser], that any CR-manifold is locally equivalent to a hypersurface in $\mathbf{C}^{n+1}$. Thus, the abstract definition given above allows for a wider class of examples only in the $C^{\infty}$-category. For a fuller discussion of this point, the reader should consult [Nirenberg].

By abuse of language, we will often say that $M$ is a CR-manifold, the Pfaffian system $I \subseteq \Omega_{\mathbf{C}}^{1}(M)$ being understood.

If $U \subseteq M$ is an open set, we say that a collection of forms $\alpha=\left(\alpha^{0}, \ldots, \alpha^{n}\right)$ in $\Omega_{\mathbf{C}}^{1}(U)$ is an adapted basis of $I$ in $U$ if

(a) $\alpha^{0}, \ldots, \alpha^{n} \operatorname{span} I$ restricted to $U$,

(b) $\alpha^{0}=\bar{\alpha}^{0}$, i.e., $\alpha^{0}$ is a real basis of $I \cap \bar{I}$ restricted to $U$.

It is immediate that $M$ is covered by open sets $U$ which possess adapted bases. If $\alpha$ and $\beta$ are two adapted bases on $U \subseteq M$, then

$$
\alpha^{0}=\lambda \beta^{0}, \quad \alpha^{i}=v^{i} \beta^{0}+g_{j}^{i} \beta^{j}, \quad i=1, \ldots, n,
$$

where $\lambda$ is a nonzero real function on $U$ and $v^{i}, g_{j}^{i} \in C_{\mathbf{C}}^{\infty}(U)$ are such that $\operatorname{det}\left(g_{j}^{i}\right) \neq 0$. From this transformation law, it follows that if we fix $x \in U$, the 
subspace $H_{x}=\operatorname{ker} \alpha_{x}^{0} \subseteq T_{x} M$ does not depend on the choice of adapted basis $\alpha$ (or on $U$, for that matter). Moreover, the map $\left[\alpha_{x}\right]: H_{x} \rightarrow \mathbf{C}^{n}$ given by

$$
\left[\alpha_{x}\right](v)=\left(\alpha_{x}^{1}(v), \ldots, \alpha_{x}^{n}(v)\right)
$$

for all $v \in H_{x}$ is an isomorphism which induces a complex structure $J_{x}$ on $H_{x}$ independent of our choice of $\alpha$ or $U$ containing $x$. The vector bundle

$$
H=\bigcup_{x \in M} H_{x} \subseteq T M
$$

together with its complex structure

$$
J=\bigcup_{x \in M} J_{x}
$$

is often referred to as the holomorphic tangent bundle of $M$.

Definition 1.2. If $M$ is a CR-manifold, a holomorphic immersion into $M$ is an immersion $\phi: N^{2 p} \rightarrow M$ with the property that, for every $y$ in $N, \phi_{*}\left(T_{y} N\right)$ is a (complex) $p$-dimensional subspace of $H_{\phi(y)}$. When $p=1$, a holomorphic immersion is called a holomorphic curve in $\boldsymbol{M}$.

REMARK. When $M$ is a real hypersurface in $\mathbf{C}^{n+1}$, a holomorphic immersion into $M$ is just a complex submanifold of $\mathbf{C}^{n+1}$ which happens to lie in $M$. This is the motivation for the above nomenclature in the abstract setting.

A fundamental observation concerning the holomorphic immersions into $M$ is the following: If $U \subseteq M$ is an open set, $\alpha$ is an adapted basis on $U$, and $\phi: N^{2 p} \rightarrow U \subseteq M$ is a holomorphic immersion, then $\phi^{*} \alpha^{0}=0$. This is clear because

$$
\phi_{*}\left(T_{y} N\right) \subseteq H_{\phi(y)}=\operatorname{ker} \alpha_{\phi(y)}^{0} .
$$

This suggests studying the structure of $\alpha^{0}$ as a 1-form. Since

$$
d \alpha^{0} \equiv 0 \bmod I_{U}=\left\{\alpha^{0}, \ldots, \alpha^{n}\right\},
$$

it follows that there exist forms $\kappa_{k}(k=1, \ldots, n)$ so that

$$
d \alpha^{0} \equiv \kappa_{k} \wedge \alpha^{k} \bmod \alpha^{0} .
$$

(Here and elsewhere, we use the summation convention.) The reality condition $\alpha^{0}=\bar{\alpha}^{0}$ implies that we may assume

$$
\kappa_{k} \equiv-i h_{k j} \overline{\alpha^{j}} \bmod \alpha^{0}
$$

so that

$$
d \alpha^{0} \equiv i h_{k j} \alpha^{k} \wedge \overline{\alpha^{j}} \quad \bmod \alpha^{0}
$$

where $\overline{h_{k j}}=h_{j \bar{k}}$.

DefinITION 1.3. The CR-manifold $M$ is said to be

(a) nondegenerate if at every $x \in M$, the matrix $h_{j \bar{k}}$ (which is hermitian) is nonsingular, i.e., $\operatorname{det}\left(h_{j k}\right) \neq 0$,

(b) strictly pseudo-convex if at every $x \in M$, the matrix $h_{j k}$ is positive or negative definite, 
(c) Lorentzian if at every $x \in M$, the matrix $h_{j k}$ has $(n-1)$ eigenvalues of the same sign and 1 eigenvalue of the opposite sign. (In particular, if $M$ is Lorentzian, then $n \geqslant 2$ and $M$ is nondegenerate.)

REMARK. It is not difficult to verify that the above definitions do not depend on the adapted basis implicit in the statement. For example, nondegeneracy is clearly equivalent to the condition $\alpha^{0} \wedge\left(d \alpha^{0}\right)^{n} \neq 0$ for the adapted basis $\alpha$ on $U$. If $\beta$ were another adapted basis on $U$, then $\beta^{0}=\lambda \alpha^{0}$ where $\lambda \neq 0$ is a real smooth function on $U$, but then

$$
\beta^{0} \wedge\left(d \beta^{0}\right)^{n}=\lambda^{n+1} \alpha^{0} \wedge\left(d \alpha^{0}\right)^{n} \neq 0 .
$$

Returning to the holomorphic immersion $\phi: N^{2 p} \rightarrow U \subseteq M$ with adapted basis $\alpha$, we see that differentiating the condition $\phi^{*} \alpha^{0}=0$ gives

$$
\phi^{*}\left(d \alpha^{0}\right)=\phi^{*}\left(i h_{k j} \alpha^{k} \wedge \alpha^{j}\right)=0 .
$$

This gives us the classical proposition

Proposition 1.4. If $\phi: N^{2 p} \rightarrow M$ is a holomorphic immersion, then for every $y$ in $N$, $\phi_{*}\left(T_{y} N\right)$ is a complex isotropic (i.e., null) space of the Levi form, i.e., the (hermitian) form $\mathcal{L}: H \times H \rightarrow \mathrm{C}$ given on an open set $U \subseteq M$ with adapted basis $\alpha$ by the formula

$$
\mathcal{L}_{x}(v, w)=h_{k \bar{j}} \alpha^{k}(v) \alpha^{\bar{j}}(w)
$$

when $v, w \in H_{x}$ for some $x \in U$. In particular, if $M$ is strictly pseudo-convex, then the only holomorphic immersions into $M$ satisfy $p=0$ (in other words, $N$ consists of isolated points). If $M$ is Lorentzian, then $M$ has no holomorphic immersions with $p>1$.

EXAMPLE 1.5. On $\mathbf{C}^{n+1}$ with coordinates $z^{0}, \ldots, z^{n}$, we let $\rho: \mathbf{C}^{n+1} \rightarrow \mathbf{R}$ be the function

$$
\rho=1+\left|z^{0}\right|^{2}-\left|z^{1}\right|^{2}-\cdots-\left|z^{n}\right|^{2} .
$$

It is easily verified that $M^{2 n+1}=\rho^{-1}(0)$ is a Lorentzian CR-manifold. Moreover, if $\left(\mu^{k}\right),\left(\lambda^{k}\right) \in \mathbf{C}^{n}$ are fixed vectors such that $\mu^{k} \overline{\mu^{k}}=\lambda^{k} \overline{\lambda^{k}}=1, \mu^{k} \overline{\lambda^{k}}=0$, then the complex line $z^{\alpha}(t)(t \in \mathbf{C})$ given by $z^{0}(t)=t, z^{k}(t)=\mu^{k}+\lambda^{k} t$ lies in $M^{2 n+1}$. This exhibits a $(4 n-4)$ (real) parameter family of holomorphic curves in $M$. We will see later that any connected holomorphic curve (i.e., $N$ is connected) in $M$ is an open subset of one of the lines given above.

To the author's knowledge, the first study of this type of example was made by [Sommer] in the case $N=2$ (the first nontrivial case). Sommer made a study of real hypersurfaces in $\mathbf{C}^{3}$ of Lorentzian type and gave several interesting examples of such hypersurfaces which possess holomorphic curves (and some which do not).

From now on, we will mainly be concerned with the Lorentzian case. Our main goal is Theorem 3.11, which asserts that, in a certain sense, the holomorphic curves in a Lorentzian CR-manifold depend on at most $n^{2}$ real parameters. Moreover, in the generic case, $M$ will have no holomorphic curves at all. These results will depend on rather high orders of "derivatives" of the CR-structure on $M$. In $\S 4$, we relate these computations to the Chern-Moser theory in the case $n=2$ to show that if $M$ has a $2^{2}=4$ parameter family of holomorphic curves, then $M$ is locally the hyperquadric (case $n=2$ in Example 1.5). 
2. The first prolongation and the canonical Pfaffian system. Throughout this section, $(M, I)$ will denote a Lorentzian CR-manifold $(n \geqslant 2), U \subseteq M$ will be an open set, and $\alpha=\left(\alpha^{0}, \ldots, \alpha^{n}\right)$ will be an adapted basis of $I$ on $U$ which satisfies

$$
d \alpha^{0} \equiv i\left(\alpha^{1} \wedge \overline{\alpha^{1}}-\alpha^{2} \wedge \overline{\alpha^{2}}-\cdots-\alpha^{n} \wedge \overline{\alpha^{n}}\right) \quad \bmod \alpha^{0} .
$$

As we have remarked before, the only possible holomorphic submanifolds of $M$ are the holomorphic curves. We will begin our study of the holomorphic curves of $M$ by analyzing the possible tangent spaces.

Let $\&_{x}$ be the set of complex 1-dimensional subspaces $\xi \subseteq H_{x}$ which are null lines for the Levi form $\mathcal{L}_{x}$. \& is easily seen to be a $S^{2 n-3}$. Set $\&=\cup_{x \in M} \&_{x}$.

$\&$ is, in a natural way, a manifold and the natural projection $\pi: \& \rightarrow M$ given by $\pi\left(\&_{x}\right)=x$ makes $\&$ into a fiber bundle over $M$ with typical fiber $S^{2 n-3}$ (see below for details). If $\phi: N^{2} \rightarrow M$ is a holomorphic curve, there is a natural map $\tilde{\phi}: N^{2} \rightarrow \&$ given by $\tilde{\phi}(y)=\phi_{*}\left(T_{y} N\right)$. \& carries an additional piece of information which allows us to characterize these maps $\tilde{\phi}: N^{2} \rightarrow \&$ which come from holomorphic curves $\phi$ : $N^{2} \rightarrow M$. If $\xi \in \&$, let $x=\pi(\xi)$. Then $\xi \subseteq H_{x} \subseteq T_{x} M$. Let $\xi^{\perp} \subseteq T_{x, \mathrm{C}}^{*} M$ be the subspace of complex 1-forms which vanish on $\xi$. Set

$$
J_{\xi}=\pi_{\xi}^{*}\left(\xi^{\perp}\right) \subseteq T_{\xi, \mathrm{C}}^{*} \& \text { and } J=\bigcup_{\xi \in \&} J_{\xi} .
$$

$J$ is a (complex) subbundle of $T_{\mathbf{C}}^{*} \&$ which is closed under conjugation, $J=\bar{J}$. We let $q$ denote the set of complex valued one-forms $\omega$ on $\&$ which satisfy $\omega_{\xi} \in J_{\xi} . g$ is a complex Pfaffian system of (complex) rank $2 n-1$. The importance of $q$ is revealed by the following proposition.

Proposition 2.1. Let $\phi: N^{2} \rightarrow M$ be a holomorphic curve and let $\tilde{\phi}: N \rightarrow \&$ be its canonical lift. Then

$$
\tilde{\phi}^{*} \omega=0 \text { for all } \omega \in \mathcal{g} .
$$

Conversely, suppose that $\tilde{\phi}: N \rightarrow \&$ satisfies (1) and that $\pi \circ \tilde{\phi}: N \rightarrow M$ is an immersion. Then $\pi \circ \tilde{\phi}: N \rightarrow M$ is a holomorphic curve in $M$ and $\tilde{\phi}$ is its canonical lift.

PROof. This is an exercise in making sure we have defined everything properly. Suppose that $\phi: N^{2} \rightarrow M$ were a holomorphic curve and that $\tilde{\phi}: N^{2} \rightarrow \&$ were its canonical lift. Let $\phi(y)=x$ and $\tilde{\phi}(y)=\xi=\phi_{*}\left(T_{y} N\right)$. Suppose $\omega \in \mathscr{g}$ is given. Then for any $y \in N$

$$
\left(\tilde{\phi}^{*}(\omega)\right)_{\left.\right|_{y}}=\tilde{\phi}_{y}^{*}\left(\omega_{\xi}\right)=\tilde{\phi}_{y}^{*}\left(\pi_{\xi}\left(\eta_{\xi}\right)\right)=(\pi \circ \tilde{\phi})_{y}^{*}\left(\eta_{\xi}\right)=\phi_{y}^{*}\left(\eta_{\xi}\right)=0
$$

where we have chosen $\eta_{\xi} \in \xi^{\perp}$ so that $\pi_{\xi}^{*}\left(\eta_{\xi}\right)=\omega_{\xi}$, and the last equation follows from the definition of $\xi^{\perp}$ and the fact that $\phi_{*}\left(T_{y} N\right)=\xi$. This establishes our first claim.

For the second, suppose that $\tilde{\phi}: N \rightarrow \&$ is such that $\tilde{\phi}^{*} \omega=0$ for all $\omega \in \mathcal{G}$ and that $\pi \circ \tilde{\phi}: N \rightarrow M$ is an immersion. We must show that $(\pi \circ \tilde{\phi})_{*}\left(T_{y} N\right)=\tilde{\phi}(y)$ for all $y \in N$. Let $\tilde{\phi}(y)=\xi$. Since $(\pi \circ \tilde{\phi})_{*}\left(T_{y} N\right)$ is of (real) dimension 2 by hypothesis, it suffices to show that $(\pi \circ \tilde{\phi})_{y}^{*}\left(\xi^{\perp}\right)=0$. But

$$
(\pi \circ \tilde{\phi})_{y}^{*}\left(\xi^{\perp}\right)=\tilde{\phi}_{y}^{*}\left(\pi_{\xi}^{*}\left(\xi^{\perp}\right)\right)=\tilde{\phi}_{y}^{*}\left(J_{\xi}\right)=0,
$$

where the last equality follows by hypothesis. Q.E.D. 
REMARK. This construction and the constructions to follow in $\$ 3$ are special cases of the technique of prolongation. $\mathcal{F}$ is called the canonical system on \& and the condition that $\pi \circ \tilde{\phi}: N \rightarrow M$ be an immersion is called the independence condition or the admissibility condition (see below).

In order to better understand the structure of $q$ we consider $g$ on $\pi^{-1}(U) \subseteq \&$. We can use $\alpha$ to exhibit an explicit diffeomorphism $A: \pi^{-1}(U) \rightarrow U \times S^{2 n-3}$. If $x \in U$ and $\xi \in \&_{x}$, then $\xi^{\perp}$ is spanned (over $\mathbf{C}$ ) by the forms

$$
\begin{aligned}
& \theta_{x}^{0}=\alpha_{x}^{0}, \\
& \theta_{x}^{k}=\alpha_{x}^{k}-\lambda^{k}(\xi) \alpha_{x}^{1}, \quad k \geqslant 2, \\
& \overline{\theta_{x}^{k}}=\overline{\alpha_{x}^{k}}-\overline{\lambda^{k}}(\xi) \overline{\alpha_{x}^{1}}, \quad k \geqslant 2,
\end{aligned}
$$

where $\lambda^{k}(\xi) \overline{\lambda^{k}(\xi)}=1$ (sum over $k \geqslant 2$ ). We define

$$
A(\xi)=\left(\pi(\xi), \lambda^{k}(\xi)\right) \in U \times S^{2 n-3} .
$$

It is immediate that $A$ is a diffeomorphism. (Or, if the reader prefers, $A$ is clearly a bijection of sets and the "natural differential structure on $\&$ " referred to earlier is the one which makes the maps $A$ diffeomorphism as $(U, \alpha)$ varies over all possible choices. Details are left to the reader.) The forms $\theta^{0}, \theta^{k}, \theta^{k}$ defined by the above equations at a point $\left(x, \lambda^{k}\right)$ in $U \times S^{2 n-3}$ are smooth complex-valued forms which pullback under $A$ to be a basis of $\mathcal{G}$ restricted to $\pi^{-1}(U)$. We could have defined $g$ locally this way, but then its universal character would have been somewhat obscured.

If $\phi: N^{2} \rightarrow M$ is a holomorphic curve, then the above proposition shows that

$$
(A \circ \tilde{\phi}) * \theta^{k}=0 \text { for all } k=0,2,3, \ldots, n
$$

moreover, since $\phi$ is an immersion, one gets

$$
\Omega=(A \tilde{\phi}) *\left(i \alpha^{1} \wedge \overline{\alpha^{1}}\right) \neq 0 .
$$

In fact, $\Omega$ is a (real) volume form on $N^{2}$. Conversely, if $\psi: N^{2} \rightarrow U \times S^{2 n-3}$ satisfies

(a) $\psi^{*} \theta^{k}=0$ for $k=0,2, \ldots, n$ and

(b) $\psi^{*}\left(i \alpha^{1} \wedge \overline{\alpha^{1}}\right) \neq 0$

then $\pi \circ \psi: N^{2} \rightarrow U \subseteq M$ is a holomorphic curve for which $\psi=A \circ(\pi \circ \psi)$. (That $\pi \circ \psi: N \rightarrow U$ is an immersion follows from (b).) This indicates how we can test maps $\psi: N^{2} \rightarrow \&$ for the property of being canonical lifts of holomorphic curves. We let $\mathscr{B} \subseteq \Omega_{\mathrm{C}}^{1} \&$ be the set of semibasic forms for the projection $\pi: \& \rightarrow M$. In $\pi^{-1}(U)$, $\mathscr{B}$ is generated by the forms $\left\{\pi^{*} \alpha^{0}, \pi^{*} \alpha^{k}, \pi^{*} \alpha^{k}\right\}$. It is visible that $\mathscr{B}$ is a complex Pfaffian system of (complex) rank $2 n+1$, that $\mathscr{B}=\overline{\mathscr{B}}$, and that $\mathcal{G} \subseteq \mathscr{B}$. Moreover, $\mathscr{B}$ is differentially closed.

DEFinition 2.2. A smooth map $\psi: N^{2} \rightarrow \&$ is said to be an integral of $q$ if $\psi^{*} \omega=0$ for all $\omega \in \mathcal{g}$. An integral $\psi: N^{2} \rightarrow \&$ of $\mathcal{G}$ is said to be admissible if, for every $y \in N$, there is a neighborhood $V$ of $y$ in $N$ and a form $\omega \in \mathscr{B}$ so that $\psi^{*}(i \omega \wedge \bar{\omega})$ is a volume form on $V$.

This rephrasing of the condition that $\pi \circ \psi: N \rightarrow M$ be an immersion is fundamental to our later work, so we restate Proposition 2.1 in this language: 
Proposition 2.1*. If $\phi: N^{2} \rightarrow M$ is a holomorphic curve in $M$, then $\tilde{\phi}: N^{2} \rightarrow \&$ is an admissible integral of $\mathcal{G}$. If $\psi: N^{2} \rightarrow \&$ is an admissible integral of $\mathcal{G}$, then $\pi \circ \psi$ : $N \rightarrow M$ is a holomorphic curve and $\psi=\overparen{\pi \circ \psi}$.

The reader who is familiar with Cartan-Kähler theory will recognize this as the classical setup for proving the existence of admissible integrals (in the analytic category), providing that the system is involutive. For a full discussion of these concepts, the reader should consult [Bryant-Chern-Griffiths]. Suffice it to say that the system $\mathscr{g}$ with independence condition $\mathscr{B}$ is not involutive, so the Cartan-Kähler theorem does not apply anyway. The standard procedure in the noninvolutive case is to "prolong" the system until it either becomes involutive or becomes "incompatible", meaning that the system has no integrals because there are no admissible tangent spaces. We will actually use a modification of the standard prolongation procedure which terminates after at most $(n-1)$ steps (see §3).

3. Systems of type $(m, k)$ and their two prolongations. In order to make progress in the iterative prolongation scheme (to be discussed below), we must have an abstract theorem which allows us to study Pfaffian systems which satisfy certain (very stringent) structure conditions.

Definition 3.1. A Pfaffian system $G \subseteq \Omega_{\mathbf{C}}^{1}(X)$ with independence bundle $\mathscr{K} \subseteq$ $\Omega_{\mathbf{C}}^{1}(X)$ is said to be of type $(m, k)$ if, for every $x \in X$, there exist

(a) an open neighborhood $U$ of $x$ with submersions

$$
\pi_{1}: U \rightarrow \mathbf{R}^{d}(d=m+2(k+1)), \quad \pi_{2}: U \rightarrow S^{2 k-1} \subseteq \mathbf{C}^{k},
$$

(b) a (complex) coframing of $\mathbf{R}^{d}$

$$
\eta^{1}, \ldots, \eta^{m}, \beta^{1}, \ldots, \beta^{k}, \overline{\beta^{1}}, \ldots, \overline{\beta^{k}}, \omega, \bar{\omega} \text {, and }
$$

(c) linear coordinates $\lambda^{1}, \ldots, \lambda^{k}$ on $\mathbf{C}^{k}$ so that $S^{2 k-1}=\left\{\left(\lambda^{\sigma}\right) \mid \lambda^{\sigma} \overline{\lambda^{\sigma}}=1\right\}$ which satisfy

(1) $\pi_{1} \times \pi_{2}: U \rightarrow \mathbf{R}^{d} \times S^{2 k-1}$ is a diffeomorphism,

(2) the forms

$$
\begin{aligned}
& \eta^{1}, \ldots, \eta^{m}, \\
& \theta^{\sigma}=\beta^{\sigma}-\lambda^{\sigma} \omega, \quad \sigma=1, \ldots, k, \\
& \overline{\theta^{\sigma}}=\overline{\beta^{\sigma}}-\overline{\lambda^{\sigma} \omega}, \quad \sigma=1, \ldots, k,
\end{aligned}
$$

are a $C_{\mathbf{C}}^{\infty}(U)$-basis for $\Phi_{\left.\right|_{v}}$,

(3) the forms $\eta^{1}, \ldots, \eta^{m}, \beta^{1}, \ldots, \beta^{k}, \overline{\beta^{1}}, \ldots, \overline{\beta^{k}}, \omega, \bar{\omega}$ are a $C_{\mathbf{C}}^{\infty}(U)$-basis of $\mathscr{K}_{\varphi_{v}}$, and

(4) $d \eta^{\mu} \equiv 0 \bmod \mathscr{q},(\mu=1, \ldots, m)$ and the span of the $\eta^{\mu}$ is closed under conjugation.

Our first remark is that for any Lorentzian CR-manifold $M^{2 n+1}$, the system $\mathscr{G} \subseteq \Omega_{\mathbf{C}}^{1}(\&)$ with independence bundle $\mathscr{B} \subseteq \Omega_{\mathbf{C}}^{1}(\&)$ is a system of type $(1, n-1)$. In fact, if we pick $\xi \in \&$, let $V \subseteq M$ be an open neighborhood of $\pi(\xi)$ diffeomorphic to $\mathbf{R}^{2 n+1}$, let $U=\pi^{-1}(V)$, and, finally, let $\alpha=\left(\alpha^{0}, \ldots, \alpha^{n}\right)$ be an adapted basis of $I$ on $V$ satisfying

$$
d \alpha^{0} \equiv i\left(\alpha^{1} \wedge \overline{\alpha^{1}}-\alpha^{2} \wedge \overline{\alpha^{2}}-\cdots-\alpha^{n} \wedge \overline{\alpha^{n}}\right) \quad \bmod \alpha^{0}
$$


then the conditions of the definition are satisfied by taking

(a) $\pi_{1} \times \pi_{2}: U \rightarrow V \times S^{2 n-3}$ to be $A: U \rightarrow V \times S^{2 n-3}$,

(b) $m=1, \eta^{1}=\alpha^{0}, k=n-1, \beta^{\sigma}=\alpha^{\sigma+1}, \sigma=1, \ldots, n-1, \omega=\alpha^{1}$,

(c) the $\lambda^{\sigma}$ as previously defined.

The only part we have not previously discussed is (4), but

$$
\begin{aligned}
d \eta^{1}=d \alpha^{0} & \equiv i\left(\alpha^{1} \wedge \overline{\alpha^{1}}-\alpha^{2} \wedge \overline{\alpha^{2}}-\cdots-\alpha^{n} \wedge \overline{\alpha^{n}}\right) \bmod \alpha^{0} \\
& \equiv i\left(\alpha^{1} \wedge \overline{\alpha^{1}}-\left(\lambda^{\sigma} \overline{\lambda^{\sigma}}\right) \alpha^{1} \wedge \overline{\alpha^{1}}\right) \bmod \mathcal{G} \\
& \equiv 0 \bmod \mathcal{G} \text {. Q.E.D. }
\end{aligned}
$$

Our second remark is that the reason for allowing for $m \neq 1$ will appear later in this section.

Our third (somewhat incidental) remark is that systems of type $(0, n)$ arise naturally when one studies the problem of imbedding a Kähler curve (Riemann surface with metric) into an arbitrary Kähler manifold of (complex) dimension $n$. Thus systems of this type have applications outside of CR-manifold theory. Moreover, systems of these types appear naturally in the study of minimal immersions of a Riemann surface into an Euclidean space of dimension $\boldsymbol{n}$. In particular, one recovers the classical fact that $X^{2}$ with metric $d s^{2}$ imbeds minimally (locally) into $\mathbf{E}^{3}$ if and only if the Gauss curvature $K$ is nonpositive and the Ricci metric $d s^{2}=$ $(-K) d \hat{s}^{2}$ has Gauss curvature 1. An analysis of this case together with some interesting results on minimal surfaces in $\mathbf{E}^{n}$ will appear elsewhere. Suffice it to say that the theorem on a finite parameter space of integrals (Theorem 3.11) applies to recover results of [Calabi] and [Lawson].

Our ultimate goal is to find a procedure for locating the admissible integrals of a Pfaffian system of type $(m, k)$. In order to do this, we study the structure of these integrals by means of the canonical coordinates furnished by Definition 3.1.

Therefore, let $X, 9, \mathcal{K}, U, \pi_{1}, \pi_{2}, \mathbf{R}^{d}, S^{2 k-1}, \eta^{\mu}, \beta^{\sigma}, \omega$ be as in Definition 3.1. We compute the exterior derivatives of the generators of $9 \bmod 9$ as follows:

$$
\begin{aligned}
& d \eta^{\mu} \equiv 0 \quad \bmod \mathscr{Q}(\text { by hypothesis), } \quad 1 \leqslant \mu \leqslant m, \\
& d \theta^{\sigma} \equiv d \beta^{\sigma}-d \lambda^{\sigma} \wedge \omega-\lambda^{\sigma} d \omega \bmod 9 \\
& \equiv-d \lambda^{\sigma} \wedge \omega+L^{\sigma} \omega \wedge \bar{\omega} \bmod \mathscr{C}
\end{aligned}
$$

for some functions $L^{\sigma}$ on $V$ (note that $L^{\sigma}$ will generally involve the $\left\{\lambda^{\sigma}\right\}$ as well as the $\mathbf{R}^{d}$ variables). Of course, we must also have

$$
d \overline{\theta^{\sigma}} \equiv-\overline{d \lambda^{\sigma}} \wedge \bar{\omega}+\overline{L^{\sigma}} \bar{\omega} \wedge \omega \bmod \varphi
$$

We compute

$$
\begin{aligned}
d\left(\overline{\lambda^{\sigma}} \theta^{\sigma}\right) & \equiv-\left(\overline{\lambda^{\sigma}} d \lambda^{\sigma}+\overline{\lambda^{\sigma}} L^{\sigma} \bar{\omega}-\lambda^{\sigma} \overline{L^{\sigma}} \omega\right) \wedge \omega \bmod \mathscr{} \\
& \equiv-\tau \wedge \omega \bmod \mathscr{}
\end{aligned}
$$

where we have set

$$
\tau=\overline{\lambda^{\sigma}} d \lambda^{\sigma}+\overline{\lambda^{\sigma}} L^{\sigma} \bar{\omega}-\lambda^{\sigma} \overline{L^{\sigma}} \omega .
$$

Note that $\tau$ satisfies $\tau+\bar{\tau}=0$, i.e., $\tau$ is purely imaginary. 
Proposition 3.2. Any admissible integral of 9 in $U, \phi: N^{2} \rightarrow U$ satisfies $\phi^{*} \tau=0$.

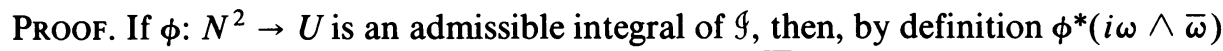
$\neq 0$ and $\phi^{*} \eta^{\mu}=\phi^{*} \theta^{\sigma}=\phi^{*} \overline{\theta^{\sigma}}=0$. It follows that $\phi^{*}\left(\overline{\lambda^{\sigma}} \theta^{\sigma}\right)=0$, so

$$
0=\phi^{*}\left(d\left(\overline{\lambda^{\sigma}} \theta^{\sigma}\right)\right)=-\phi^{*} \tau \wedge \phi^{*} \omega .
$$

Conjugating and using $\tau+\bar{\tau}=0$, we get $\phi^{*} \tau \wedge \phi^{*} \bar{\omega}=0$. Since $\phi^{*} \omega \wedge \phi^{*} \bar{\omega} \neq 0$, we immediately see that $\phi^{*} \tau=0$. Q.E.D.

From now on, we write $\mathscr{G}_{+}$for the system spanned by $\tau$ and $\mathscr{G}$. The previous proposition then says that the admissible integrals of $\Phi_{+}$are the same as the admissible integrals of $\mathcal{G}$.

Definition 3.3. A 2-dimensional subspace $\xi \subseteq T_{x} X$ (for some $x \in X$ ) will be called an admissible integral element of $\Phi_{+}$if

(1) $\alpha(v)=0$ for all $\alpha \in \mathscr{G}_{+}, v \in \xi$,

(2) $(d \alpha)_{x}(v, w)=0$ for all $\alpha \in q_{+}, v, w \in \xi$,

(3) $(i \omega \wedge \bar{\omega})(v, w) \neq 0$ if $v, w \in \xi$ are linearly independent.

We have the easy

Proposition 3.4. A smooth map $\phi: N \rightarrow X$ is an admissible integral of $\varphi$ if and only if $\phi_{*}\left(T_{y} N\right)$ is an admissible integral element of $G_{+}$for every $y \in N$.

The only subtlety is the fact that $G_{+}$is globally defined without reference to a special $U \subseteq X$, etc. This is not difficult and is left to the reader.

In order to obtain complete information about the structure of the admissible integral elements of $g_{+}$, we must compute $d \tau$. Using the defining formula for $\tau$ and the fact that $\tau+\bar{\tau}=0$, we get that there exist complex functions $A_{\sigma}, B_{\sigma}, C$ on $U$ which satisfy

$$
\begin{aligned}
d \tau \equiv & d \lambda^{\sigma} \wedge \overline{d \lambda^{\sigma}}+\left(A_{\sigma} d \lambda^{\sigma}\right) \wedge \omega-\left(\overline{A_{\sigma}} \overline{d \lambda^{\sigma}}\right) \wedge \bar{\omega} \\
& +\left(B_{\sigma} \overline{d \lambda^{\sigma}}\right) \wedge \omega-\left(\overline{B_{\sigma}} d \lambda^{\sigma}\right) \wedge \bar{\omega}+C \omega \wedge \bar{\omega} \bmod q_{+} .
\end{aligned}
$$

We recall

$$
d \theta^{\sigma} \equiv-\left(d \lambda^{\sigma}+L^{\sigma} \bar{\omega}\right) \wedge \omega \bmod \mathscr{\omega} .
$$

If $\xi \subseteq T_{x} X$ is an admissible integral element of $\mathscr{G}_{+}$we see that $\xi^{\perp} \subseteq T_{\mathbf{C}, x}^{*} X$ is spanned by forms

$$
\begin{array}{ll}
\eta^{\mu}, & \mu=1, \ldots, m, \\
\boldsymbol{\theta}^{\sigma}, \overline{\theta^{\sigma}}, & \sigma=1, \ldots, K, \\
d \lambda^{\sigma}+L^{\sigma} \bar{\omega}-\kappa^{\sigma} \omega, & \sigma=1, \ldots, K, \\
\overline{d \lambda^{\sigma}}+\overline{L^{\sigma}} \omega-\bar{\kappa}^{\sigma} \bar{\omega}, & \sigma=1, \ldots, K,
\end{array}
$$

for some complex numbers $\left(\kappa^{\sigma}\right)$. In order that $\tau \in \xi^{\perp}$, we see that we must have

$$
\overline{\lambda^{\sigma}} \kappa^{\sigma}=\lambda^{\sigma} \overline{L^{\sigma}} \text {. }
$$

In order that $d \tau$ vanish on $\xi$ while $\omega \wedge \bar{\omega}$ remains nonzero, we see that we must require

$\left(*^{*}\right) \quad\left(\kappa^{\sigma}-B_{\sigma}\right)\left(\overline{\kappa^{\sigma}-B_{\sigma}}\right)+C+\left(A_{\sigma} L^{\sigma}+\overline{A_{\sigma} L^{\sigma}}\right)+L^{\sigma} \overline{L^{\sigma}}+B_{\sigma} \overline{B_{\sigma}}=0$. 
Equation $\left({ }^{*} 1\right)$ forces $\left(\kappa^{\sigma}\right) \in \mathbf{C}^{k}$ to lie in a complex hyperplane $H_{x}^{k-1} \subseteq \mathbf{C}^{k}$, while equation $\left({ }^{*} 2\right)$ is either insatiable or it forces $\left(\kappa^{\sigma}\right) \in \mathbf{C}^{k}$ to lie in a real hypersphere $S_{x}^{2 k-1}$ centered at $B_{\sigma}$. It follows that there are three cases to be considered:

Case \#. $H_{x}^{k-1} \cap S_{x}^{2 k-1}=\varnothing$. In this case, there are no admissible integral elements $\xi \subseteq T_{x} X$ of $\mathscr{G}_{+}$. We set

$$
X^{\#}=\left\{x \in X \mid H_{x}^{k-1} \cap S_{x}^{2 k-1}=\varnothing\right\} .
$$

$X^{\#}$ is clearly an open subspace of $X$ (which may be empty). It is immediate that there are no admissible integrals of $\mathscr{G}$ in $X$ whose images intersect $X^{\#}$.

Case घ. $H_{x}^{k-1} \cap S_{x}^{2 k-1}$ consists of a single point, say $\left(\kappa^{\sigma}\right) \in \mathbf{C}^{k}$. Geometrically this means that either $k=1$ and $H_{x}^{0}$ is a point on $S_{x}^{1}$, or $k>1$ and $H_{x}^{k-1}$ is tangent to $S_{x}^{2 k-1}$ at $\left(\kappa_{x}^{\sigma}\right)$. We set

$$
X^{\natural}=\left\{x \in X \mid H_{x}^{k-1} \cap S_{x}^{2 k-1} \text { is a point in } \mathbf{C}^{k}\right\} .
$$

$X^{\natural}$ is a closed subspace of $X$ (it may be empty). At each $x \in X$, there is a unique integral element of $g_{+}$which is admissible. We denote this element by $\xi_{x} \subseteq T_{x} X$. Despite its nongeneric appearance, this case is extremely important. Later we will see that the admissible integrals of $\mathscr{G}$ which lie in $X^{\natural}$ can be found via the Frobenius test.

Case b. $H_{x}^{k-1} \cap S_{x}^{2 k-1}=S_{x}^{2 k-3} \subseteq \mathbf{C}^{k}$. This is the most interesting case. We set

$$
X^{\mathrm{b}}=\left\{x \in X \mid H_{x}^{k} \cap S_{x}^{2 k-1}=S_{x}^{2 k-3}\right\} .
$$

Every $x \in X^{\mathrm{b}}$ has a $(2 k-3)$-sphere of admissible integral elements of $\Phi_{+} \cdot$ (Notice that when $k=1$, this case is impossible. In fact $H_{x}^{0}$ will be a point in $\mathbf{C}$ and $S_{x}^{1}$ will be a circle there. The only case when $H_{x}^{0} \cap S_{x}^{1} \neq \varnothing$ is when $H_{x}^{0} \in S_{x}^{1}$, which falls in Case ต. If $H_{x}^{0} \notin S_{x}^{1}$, then $x \in X^{\#}$.)

Let $\&^{b}$ be the space of admissible integral elements $\xi \subseteq T_{x} X$ as $x$ ranges over $X^{b}$. The natural projection $\pi: \&^{b} \rightarrow X^{b}$ is a surjection and its fiber at every point $x \in X^{b}$ is diffeomorphic to $S^{2 k-3}$. In fact, it is not hard to prove that $\&^{b}$ is a fiber bundle over $X^{b}$ with the natural topology and differentiable structure. Moreover, $\&^{b}$ has a canonical system $\mathscr{G}^{b} \subseteq \Omega_{\mathbf{C}}^{1} \&^{b}$ with independence bundle $\mathscr{K}^{b} \subseteq \Omega_{\mathbf{C}}^{1} \&^{b}$ defined as follows: If $\xi \in \&^{b}$ is given, let $\pi(\xi)=x \in X^{b}$ and let $\xi^{\perp} \subseteq T_{x, C}^{*} X^{b}$ be the annihilator of $\xi \subseteq T_{x} X^{b}$. Define

$$
I_{\xi}^{b}=\pi_{\xi}^{*}\left(\xi^{\perp}\right) \subseteq T_{\xi, \mathrm{C}}^{*} \&^{b}, \quad K_{\xi}^{b}=\pi_{\xi}^{*}\left(T_{x, \mathrm{C}} X^{b}\right) \subseteq T_{\xi, \mathrm{C}}^{*} \&^{b}
$$

and $I^{b}=\cup_{\xi} I_{\xi}^{b}, K^{b}=\cup_{\xi} K_{\xi}^{b} . I^{b}$ and $K^{b}$ are smooth complex subbundles of $T_{C}^{*} \&^{b}$. We define

$$
g^{b}=\left\{\omega \in \Omega_{\mathbf{C}}^{l}\left(\&^{b}\right) \mid \omega_{\xi} \in I_{\xi}^{b}\right\} \quad \text { and } \quad \mathscr{K}^{b}=\left\{\omega \in \Omega_{\mathbf{C}}^{l}\left(\&^{b}\right) \mid \omega_{\xi} \in \mathscr{K}_{\xi}^{b}\right\} .
$$

Given any admissible integral $\phi: N^{2} \rightarrow X^{\mathrm{b}}$ of $\varphi$, there is the canonical lift $\tilde{\phi}$ : $N \rightarrow \&^{b}$

$$
\tilde{\phi}(y)=\phi_{*}\left(T_{y} N\right) .
$$

We then have the following proposition analogous to Proposition $2.1^{*}$ and whose proof is so similar as to be omitted. 
Proposition 3.5. If $\phi: N^{2} \rightarrow X^{b}$ is an admissible integral of 9 , then $\phi: N \rightarrow \&^{b}$ is an admissible integral of $\mathrm{g}^{\mathrm{b}}$. If $\psi: N \rightarrow \&^{\mathrm{b}}$ is an admissible integral of $\mathrm{g}^{\mathrm{b}}$, then $\pi \circ \psi$ : $N \rightarrow X$ is an admissible integral of $\mathscr{G}$ and, moreover $\psi=\widetilde{\pi \circ \psi}$.

The next major tool in our procedure is the completion of the "induction step".

THeOREM 3.6. If $\mathscr{G} \subseteq \Omega_{\mathbf{C}}^{1} X$ with independence bundle $\mathscr{K} \subseteq \Omega_{\mathbf{C}}^{1}(X)$ is a Pfaffian system of type $(m, k)$, and, in addition, $X^{b} \neq \varnothing$, then $g^{b} \subseteq \Omega_{C}^{1} \&^{b}$ with independence bundle $\mathscr{K}^{b} \subseteq \Omega_{\mathrm{C}}^{1} \&^{b}$ is a Pfaffian system of type $(m+2 k+1, k-1)$.

Proof. The details are somewhat messy, but we give them because of the importance of the technique. We keep the same notations as before, but now we assume $U \subseteq X^{\text {b }}$. Let $\left(h_{x}^{\sigma}\right) \in \mathbf{C}^{k}$ be the center of $S_{x}^{2 k-3} \subseteq \mathbf{C}^{k}$, and let $r_{x}>0$ be its radius. Both $\left(h_{x}^{\sigma}\right)$ and $r_{x}$ are smooth functions on $U \subseteq X$.

If $\xi \in \&$ is such that $\pi(\xi)=x \in U$, then $\xi^{\perp}$ is spanned by $\eta^{\mu}, \mu=1, \ldots, m, \theta^{\sigma}$, $\overline{\theta^{\sigma}}, \beta^{\sigma}=d \lambda^{\sigma}+L^{\sigma} \bar{\omega}-\kappa^{\sigma}(\xi) \omega$, and $\overline{\beta^{\sigma}}$, where $\kappa^{\sigma}(\xi)$ satisfies $\left({ }^{*} 1\right)$ and $\left({ }^{*} 2\right)$ above. In other words, $\kappa^{\sigma}(\xi)=h_{x}^{\sigma}+r_{x} \tau^{\sigma}(\xi)$ where $\tau^{\sigma} \overline{\tau^{\sigma}}=1$ and $\tau^{\sigma} \overline{\lambda^{\sigma}}=0$. Defining $\gamma^{\sigma}=d \lambda^{\sigma}$ $+L^{\sigma} \bar{\omega}-h^{\sigma} \omega$, we have $\beta^{\sigma}=\gamma^{\sigma}-r \tau^{\sigma}(\xi) \omega$. Let $\left(g_{\rho}^{\sigma}\right)$ be a unitary matrix-valued function on $U$ which satisfies

$$
g_{\rho}^{k}=\overline{\lambda^{\rho}}
$$

(we may have to shrink $U$ to do this). Set

$$
\alpha^{\sigma}=r^{-1} g_{\rho}^{\sigma} \beta^{\rho}=r^{-1} g_{\rho}^{\sigma} \gamma^{\rho}-g_{\rho}^{\sigma} \tau^{\rho}(\xi) \omega .
$$

Define $\eta^{\sigma}=r^{-1} g_{\rho}^{\sigma} \gamma^{\rho}$ and $t^{\sigma}(\xi)=g_{\rho}^{\sigma} \tau^{\rho}(\xi)$. Then $t^{k}(\xi)=0$ and $t^{\sigma}(\xi) \bar{t}^{\sigma}(\xi)=1$ because of the unitary nature of the matrix $g$. We easily compute that

$$
\alpha^{k}=\eta^{k}=r^{-1} \tau
$$

To sum up, $\xi^{\perp}$ has a basis given by

$$
\xi^{\perp} \begin{cases}\eta^{\mu}, & \mu=1, \ldots, m, \\ \theta^{\sigma}, \overline{\theta^{\sigma}}, & \sigma=1, \ldots, K, \\ \tau, & \sigma=1, \ldots, k-1, \\ \alpha^{\sigma}=\eta^{\sigma}-t^{\sigma}(\xi) \omega, & \\ \hline \alpha^{\sigma} & \end{cases}
$$

We take these forms as generators of 9 on $\pi^{-1}(U) \subseteq \&$. The map $A: \pi^{-1}(U) \rightarrow U$ $\times S^{2 k-3}$ given by $A(\xi)=\left(\pi(\xi), t^{\circ}(\xi)\right), 1 \leqslant \sigma \leqslant k-1$, yields the new $\pi_{1}$ and $\pi_{2}$. The complex coframing of $U$ (which we may assume is $\simeq \mathbf{R}^{d}$ )

$$
\underbrace{\eta^{\mu}, \theta^{\sigma}, \overline{\theta^{\sigma}}, \tau}_{m+2 k+1} ; \underbrace{\alpha^{\sigma}, \overline{\alpha^{\sigma}}}_{2(k-1)} ; \underbrace{\omega, \bar{\omega}}_{2}
$$

and the coordinates $t^{\sigma}(\xi)$ on $C^{k-1}$ clearly satisfy (1)-(3) of Definition 3.1. It remains to verify (4).

$$
d \theta^{\sigma} \equiv-\beta^{\sigma} \wedge \omega \equiv 0 \bmod \varphi
$$


because $\beta^{\sigma}$ (and $\overline{\beta^{\sigma}}$ ) belong to 9 . Similarly $d \overline{\theta^{\sigma}} \equiv 0 \bmod \mathscr{~}$. It remains to verify that $d \tau \equiv 0 \bmod 9$. But the relation $\left({ }^{*} 2\right)$ was chosen just so that this would be true. Q.E.D.

With this inductive step in mind, we make the following definition.

Definition 3.7. Let $\mathscr{G} \subseteq \mathcal{K} \subseteq \Omega_{\mathbf{C}}^{1}(X)$ be a Pfaffian system (with independence bundle) of type $(m, k)$. We set $X_{0}=X=X_{0}^{\#} \cup X_{0}^{\natural} \cup X_{0}^{b}, g^{(0)}=q, K^{(0)}=\mathscr{K}$, and inductively define, for $0<j \leqslant j_{0}$,

$$
\begin{gathered}
X_{j}=\&^{\mathrm{b}}\left(X_{j-1}^{\mathrm{b}}\right)=X_{j}^{\#} \cup X_{j}^{\natural} \cup X_{j}^{\mathrm{b}}, \\
g^{(j)}=\left(\mathscr{G}^{(j-1)}\right)^{b}, \quad \mathscr{K}^{(j)}=\left(\mathcal{K}^{(j-1)}\right)^{\mathrm{b}},
\end{gathered}
$$

where $j_{0}$ is the smallest integer for which $X_{j_{0}}^{b}=\varnothing$. Note that $j_{0}<k$ because $g^{(j)} \subseteq \mathscr{K}^{(j)} \subseteq \Omega_{\mathbf{C}}^{1}\left(X_{j}\right)$ is a Pfaffian system (with independence bundle) of type $\left(m_{k, j}, k-j\right)$ and $X^{b}=0$ for a system $9 \subseteq \mathscr{K} \subseteq \Omega_{\mathbf{C}}^{1}(X)$ of type $(m, 1)$.

Note $m_{k, j}=m+j(2 k+2-j)$.

We remark that $X_{j}$ is a smooth manifold of dimension $d_{j}=m+(j+2)(2 k-j)$ +1 (if it is nonempty). Moreover $X_{j}^{\natural} \subseteq X_{j}$ is a closed subspace. In general it will have singularities.

Suppose we are given an admissible integral $\phi: N \rightarrow X=X_{0}$. We know already that $\phi(N) \cap X_{0}^{\#}=\varnothing$. We let $K_{0}=\phi^{-1}\left(X_{0}^{\natural}\right)$ and set $N_{1}=N \backslash K_{0} . K_{0}$ is a closed set since $X_{0}^{\natural} \subseteq X_{0}$ is closed. If $N_{1} \neq \varnothing$, then $\phi\left(N_{1}\right) \subseteq X_{0}^{b}$ and there is a canonical lift $\tilde{\phi}$ : $N_{1} \rightarrow X_{1}=\&^{b}\left(X_{0}^{b}\right)$ so that $\tilde{\phi}$ is an admissible integral of $g^{(1)}$. We denote this lift by $\phi^{1}: N_{1} \rightarrow X_{1}$. By induction, we define $K_{j}=\left(\phi^{j}\right)^{-1}\left(X_{j}^{\natural}\right)$ and set $N_{j+1}=N_{j} \backslash K_{j}$. If $N_{j+1} \neq \varnothing$, we let $\phi^{j+1}: N_{j+1} \rightarrow X_{j+1}=\&^{b}\left(X_{j}^{b}\right)$ be the canonical lift of $\phi^{j}: N_{j+1} \rightarrow X_{j}$. We note that $K_{j} \subseteq N_{j}$ is a closed subspace.

Definition 3.8. The osculation degree of $\phi: N \rightarrow X$ is the smallest integer $\delta_{\phi}$ such that $N_{\delta_{\phi}}=\varnothing$, (we always have $1 \leqslant \delta_{\phi} \leqslant j_{0}+1$ ).

REMARK. The terminology is chosen so as to suggest the degree of osculation of a curve in $\mathbf{C}^{n}$. In fact, for the differential system governing the metric embedding of a holomorphic curve into $\mathbf{C}^{n}$, the two degrees are the same.

If we let $U_{j} \subseteq K_{j}$ be the interior of $K_{j}$ as a subspace of $N$, the map $\phi^{j}$ : $U_{j} \rightarrow X_{j}^{\natural} \subseteq X_{j}$ is an admissible integral of $g^{(j)}$ when $U_{j} \neq \varnothing$. Since the union of the $U_{j}\left(0 \leqslant j \leqslant \delta_{\phi}\right)$ is dense and open in $N$, we see that we may find the "essential pieces" of any holomorphic curve $\phi: N \rightarrow X$ by finding the admissible integrals of $g^{(j)}$ which lie in $X_{j}^{\mathrm{q}}$ for all $j\left(0 \leqslant j \leqslant j_{0}\right)$.

Let us say that $\phi: N \rightarrow X$ has constant osculation if $K_{j}=\varnothing$ for $j<\delta_{\phi}-1$. Thus $U_{\delta_{\phi}-1}=K_{\delta_{\phi}-1}=N$. Our assertion then can be expressed as

ObSERVATION. For any $\phi: N \rightarrow X$ which is an admissible integral of $\mathcal{Q}, N$ contains a dense open set $U$ with a decomposition $U=U_{0} \cup U_{1} \cup \cdots \cup U_{\delta_{\phi}-1}$ into disjoint open sets such that $\phi: U_{j} \rightarrow X$ is of constant osculation.

From now on, we concentrate on curves of constant osculation. If $\phi: N \rightarrow X$ is such a curve of osculation degree $j+1$, then $\phi: N \rightarrow X$ has a canonical lift $\tilde{\phi}$ : $N \rightarrow X_{j}$ so that $\tilde{\phi}(N) \subseteq X_{j}^{\natural}$ and $\tilde{\phi}: N \rightarrow X_{j}$ is an admissible integral of $g^{(j)}$. Our next theorem shows that these integrals are characterized by a system of corank 2 (the largest possible). 
TheOREM 3.9. Suppose that $\mathscr{G} \subseteq \mathcal{K} \subseteq \Omega_{\mathbf{C}}^{1}(X)$ is a (complex) Pfaffian system (with independence condition) of type $(m, k)$ and suppose that $X_{j}^{\natural} \neq \varnothing$ for some $j \leqslant j_{0}$. Then there is a (canonical) Pfaffian system $\mathcal{L}_{j} \subseteq \Omega_{\mathbf{C}}^{1}\left(X_{j}\right)$ of corank 2 (i.e., $\mathcal{L}_{j}^{\perp}(x) \subseteq T_{x} X_{j}$ has dimension 2 for all $x \in X_{j}$ ) whose two-dimensional integrals are the admissible integrals of $\mathcal{G}^{(j)}\left(\phi: N \rightarrow X_{j}\right)$ which satisfy $\phi(N) \subseteq X_{j}^{\natural}$.

Proof. By induction, it suffices to prove the claim for $j=0$. We retain the notations and conventions of the discussion between Propositions 3.4 and 3.5. For each $x \in X=X_{0}$, we let $\left(\kappa^{\sigma}(x)\right)$ be the unique point on $H_{x}^{k-1}$ which is closest to the center of the sphere $S_{x}^{2 k-1}$, i.e., $\left(B_{\mathrm{\sigma}}\right) \in \mathbf{C}^{k}$. Explicitly,

$$
\kappa^{\sigma}=B_{\sigma}+\left(\sum_{s} \lambda^{s} \overline{L^{s}}-\overline{\lambda^{s}} B_{s}\right) \lambda^{\sigma} .
$$

The formula shows that $\kappa^{\alpha}$ is smooth (analytic) if $B_{\sigma}, L^{\sigma}$, and $\lambda^{\sigma}$ are smooth (analytic). We let $\mathcal{L}_{0}$ restricted to $U$ be generated by the forms

$$
\begin{gathered}
\eta^{\mu}, \theta^{\sigma}, \overline{\theta^{\sigma}} \quad(\mu=1, \ldots, m, \sigma=1, \ldots, k), \\
\pi^{\sigma}=d \lambda^{\sigma}+L^{\sigma} \omega-\kappa^{\sigma} \omega \quad(\sigma=1, \ldots, k),
\end{gathered}
$$

and

$$
\overline{\pi^{\sigma}} \quad(\sigma=1, \ldots, k) .
$$

$\varrho_{0}=\overline{\complement_{0}}$ is of rank $m+4 k-1$ while $X=X_{0}$ is of dimension $m+4 k+1$. Thus $\varrho_{0}$ has corank 2. We define $\xi_{x}^{2} \subseteq T_{x} X$ to be the annihilator of $\varrho_{0}(x) \subseteq T_{x, \mathbf{C}}^{*} X$. The condition that $\left(\kappa_{x}^{\sigma}\right)$ lie on $H_{x}^{k-1}$ (condition ( $\left.{ }^{*} 1\right)$ ) insures that $\tau \in \mathcal{L}_{0}$ so that, in fact $q_{+} \subseteq \mathcal{L}_{0}$. We infer that any integral of $\mathcal{L}_{0}$ of dimension 2 must also be an integral of 9. Moreover, because $\{\omega, \bar{\omega}\}$ completes $\varrho_{0}$ to span $\Omega_{\mathbf{C}}^{1}(X)$, it follows that any 2-dimensional integral of $\varrho_{0}$ is an admissible integral of 9 . Now $\xi_{x}$ is an integral element of $d \tau$ only if $\kappa^{\sigma}(x)$ satisfies condition $\left({ }^{*} 2\right)$ and this occurs only if $\kappa^{\sigma}(x) \in$ $S^{2 k-1}$ which is equivalent to $x \in X_{0}^{\natural}$ by the definition of $\kappa^{\sigma}$. Since $\tau \in \varrho_{0}$, this implies that every two-dimensional integral of $\varrho_{0}$ actually lies in $X_{0}^{\natural}$.

To finish the proof, we must verify that any admissible integral of 9 in $X_{0}^{\natural}$ is an integral of the larger system $\mathcal{L}_{0}$. Our definitions are rigged just so that $\xi_{x}$ is the unique admissible integral element of $\mathscr{q}$ when $x \in X_{0}^{\natural}$, so this is clear. Q.E.D.

Definition 3.10. If $G \subseteq \mathscr{K} \subseteq \Omega_{\mathbf{C}}^{1}(X)$ is a Pfaffian system (with independence condition) of type ( $m, k)$, we say that $X_{j}$ with the Pfaffian system $\mathcal{L}_{j} \subseteq \Omega_{\mathbf{C}}^{1}\left(X_{j}\right)$ is the Gj-prolongation of $(\mathcal{G}, \mathcal{K})$. We say that $X_{j}$ with the system $\mathscr{G}^{(j)} \subseteq \mathcal{K}^{(j)} \subseteq \Omega_{\mathbf{C}}^{\mathrm{l}}\left(X_{j}\right)$ is the bj-prolongation of $(9, \mathscr{K})$.

Since the $b k$-prolongation is always empty for a system of type $(m, k)$, the problem of finding the integrals of $G \subseteq \Omega_{\mathbf{C}}^{1}(X)$ "reduces" to the problem of finding the integrals of the corank 2 systems $\varrho_{j} \subseteq \Omega_{\mathbf{C}}^{1}\left(X_{j}\right)$ for $j=0, \ldots, j_{0}$. This is a collection of Frobenius problems and is, in principle, always solvable by ODE. For example, the simplest possible case is that $\mathcal{L}_{j_{0}}$ be differentially closed. The Frobenius theorem then asserts that $X_{j_{0}}$ is foliated by the two-dimensional integrals of $\varrho_{j_{0}}$. We then say that the integrals of $\varrho_{j_{0}}$ depend on (dim $\left.X_{j_{0}}-2\right)$ (real) parameters. 
More generally, we have the important

ObServation. If $\mathscr{G} \subseteq \mathcal{K} \subseteq \Omega_{\mathbf{C}}^{1}(X)$ is a Pfaffian system (with independence condition) of type ( $m, k)$, the admissible integrals of 9 depend on at most

$$
\begin{aligned}
\operatorname{dim} X_{j_{0}}-2 & =m+\left(j_{0}+2\right)\left(2 k-j_{0}\right)-1 \\
& \leqslant m-1+(k+1)^{2} \quad\left(\text { since } j_{0}<k\right)
\end{aligned}
$$

(real) parameters.

Which in turn gives

THEOREM 3.11. If $M^{2 n+1}$ is a Lorentzian CR-manifold then its holomorphic curves depend on at most $n^{2}$ parameters.

Proof. The system $\mathscr{f} \subseteq \mathscr{B} \subseteq \Omega_{\mathbf{C}}^{1}(\&)$ is a system of type $(1, n-1)$. The above observation shows that the holomorphic curves depend on at most

$$
1-1+((n-1)+1)^{2}=n^{2}
$$

parameters. Q.E.D.

We will give an example in $\$ 5$ to show that this upper bound can actually be attained. Moreover, in $\S 4$, we show that if $M^{5}$ has a $n^{2}=4$ parameter family of holomorphic curves (one tangent to every null vector of the Levi form), then $M^{5}$ is locally equivalent to the hypersurface

$$
M=\left\{\left.\left(X_{0}, X_{1}, X_{2}\right) \in \mathbf{C}^{3}|1+| X_{0}\right|^{2}-\left|X_{1}\right|^{2}-\left|X_{2}\right|^{2}=0\right\} .
$$

4. The case $n=2$ and the Chern-Moser theory. In this section, we relate our previous calculations for the general case to the Chern-Moser theory in the simplest possible case: $n=2$. We will maintain all of the notations of [Chern-Moser] in $\$ \S 4-5$ and its Appendix. We simplify matters by assuming (as we may, since $M^{5}$ is Lorentzian) that $g_{\alpha \beta}-$ is of the diagonal form

$$
\left(g_{\alpha \bar{\beta}}\right)=\left(\begin{array}{ll}
1 & 0 \\
0 & -1
\end{array}\right) \text {. }
$$

Let $U \subseteq M^{5}$ be such that there is a local section $\sigma: U \rightarrow Y$ of the canonical bundle $Y \rightarrow M$. We denote the pullbacks of the canonical forms on $Y$ by the same letters as represented the forms on $Y$, i.e., we write $\omega$ for $\sigma^{*} \omega$, etc. This can cause no confusion because we will never work on $Y$. The forms $\omega, \omega^{\alpha}, \overline{\omega^{\beta}}, \phi, \phi_{\alpha}^{\beta}, \phi^{\alpha}, \overline{\phi^{\beta}}, \psi$ on $M^{5}$ satisfy all the equations A.1-A.6, and the tensors $S, V, Q, P, R$ have become collections of functions on $M$.

Now $\left(\omega, \omega^{1}, \omega^{2}\right)$ is an adapted basis of $I$ on $U$ and satisfies

$$
d \omega \equiv i\left(\omega^{1} \wedge \overline{\omega^{1}}-\omega^{2} \wedge \overline{\omega^{2}}\right) \bmod \omega .
$$

If $\pi: \& \rightarrow M$ is the bundle defined in $\S 2$, then $A: \pi^{-1}(U) \rightarrow U \times S^{1}$ gives a coordinate system on $\pi^{-1}(U)$ where we use $\theta$ as a coordinate on $S^{1}$. The canonical system $\mathcal{G}$ on $U \times S^{1}$ is then generated by

$$
\left.\begin{array}{c}
\omega \\
\kappa=\omega^{2}-e^{i \theta} \omega^{1} \\
\bar{\kappa}=\overline{\omega^{2}}-e^{-i \theta} \overline{\omega^{1}}
\end{array}\right\} \mathscr{F}
$$


we clearly have $d \omega \equiv 0 \bmod \mathscr{q}$. The relations

$$
\left.\begin{array}{l}
d \kappa \equiv-e^{i \theta} \tau \wedge \omega^{1} \\
d \bar{\kappa} \equiv e^{i \theta} \tau \wedge \overline{\omega^{1}}
\end{array}\right\} \bmod \mathscr{g}
$$

where $\tau=\operatorname{id} \theta+e^{-i \theta} \phi_{1}^{2}+\left(\phi_{2}^{2}-\phi_{1}^{1}\right)-e^{i \theta} \phi_{2}^{1}$ follow by computation. Using the identities which the $\phi_{\alpha}^{\beta}$ satisfy we easily get that $\tau+\bar{\tau}=0$, so $\mathscr{q} \cup\{\tau\}$ generates $g_{+}$. To find the admissible integral elements of $g_{+}$, we must differentiate $\tau \bmod g_{+}$. After a brief computation, the answer comes out

$$
\begin{aligned}
d \tau & \equiv e^{-i \theta} \Phi_{1}^{2}+\left(\Phi_{2}^{2}-\Phi_{1}^{1}\right)-e^{i \theta} \Phi_{1}^{2} \bmod \mathcal{g}_{+} \\
& \equiv-\left[a_{2} e^{2 i \theta}+4 a_{1} e^{i \theta}+6 a_{0}+4 \bar{a}_{1} e^{-i \theta}+\bar{a}_{2} e^{-2 i \theta}\right] \omega^{1} \wedge \overline{\omega^{1}} \bmod \mathcal{F}_{+}
\end{aligned}
$$

where $a_{0}=S_{11 \overline{11}}=\bar{a}_{0}, a_{1}=S_{21 \overline{11}}, a_{2}=S_{22 \overline{11}}$ are the five (real) components of the $S$ tensor ( $a_{1}$ and $a_{2}$ are complex).

Since we are in the case $n=2$, there is no $b$-prolongation. The 4 -prolongation is just the system $g_{+}$on $\&$ (note that $g_{+}$has corank 2 and is closed under conjugation). This leads us to

THeOREM 4.1. A Lorentzian CR-manifold $M^{5}$ has a holomorphic curve tangent to every element of \& (= the space of null complex lines of the Levi form) if and only if $M^{5}$ is $C R$ equivalent locally to the hyperquadric $(n=2$ in Example 1.5).

Proof. If $M^{5}$ is locally equivalent to the hyperquadric then $M^{5}$ clearly has a holomorphic curve tangent to each element of \& (just look at Example 1.5). The other direction is more subtle.

$M^{5}$ has a holomorphic curve tangent to every element of $\&$ if and only if $g_{+} \subseteq \Omega_{\mathrm{C}}^{1} \&$ is differentially closed. Since $g_{+}$is generated in $\pi^{-1}(U) \simeq U \times S$ by $\omega, \kappa$, $\bar{\kappa}, \tau$, we compute

$$
\begin{gathered}
d \omega \equiv 0 \bmod \mathcal{F}_{+}, \quad d \kappa \equiv 0 \bmod \mathscr{g}_{+}, \\
d \tau \equiv-\left[a_{2} e^{2 i \theta}+4 a_{1} e^{i \theta}+6 a_{0}+4 \bar{a}_{1} e^{-i \theta}+\bar{a}_{2} e^{-2 i \theta}\right] \omega^{1} \wedge \overline{\omega^{1}} \bmod \mathcal{F}_{+} .
\end{gathered}
$$

The system is closed iff

$$
K(\theta)=a_{2} e^{2 i \theta}+4 a_{1} e^{i \theta}+6 a_{0}+4 a_{1} e^{-i \theta}+a_{2} e^{-2 i \theta} \equiv 0 .
$$

Since the $a_{i}$ do not depend on $\theta$, this can only happen when $a_{0} \equiv a_{1} \equiv a_{2} \equiv 0$. In other words (using the symmetry of the $S$-tensor), $M^{5}$ satisfies the hypothesis if and only if the $S$-tensor vanishes identically.

Using the Bianchi identities in the Chern-Moser Appendix, we compute that the vanishing of the $S$-tensor implies $V \equiv P \equiv Q \equiv 0$. Finally by differentiating A.1-A.6, we conclude that $R \equiv 0$. Thus all of the CR-curvature on $Y$ is identically zero and the standard theorem in $\$ 5$ of Chern-Moser shows that $M^{5}$ is locally equivalent to the hyperquadric. Q.E.D.

It would be interesting to have a direct proof of this theorem, i.e., which used the existence of the holomorphic curves to construct a CR-map from $M^{5}$ to the hyperquadric without the intervention of the Chern-Moser theory. This appears to be difficult but it might furnish some insight into the structure of the space of holomorphic curves in $M^{2 n+1}$ when $n>2$. 
In the general case, of course, the function $K(\theta)$ will not vanish identically for all values of $\theta$ at a point $x \in U$. In the classical literature the points where $K(\theta) \equiv 0$ for all $\theta$ are called umbilic points of $M$ (see [Chern-Moser]). If $x$ is not umbilic then $K_{x}(\theta)=0$ has at most 4 solutions (in $\theta$ ) and we get

Proposition 4.2. Through a nonumbilic point $x \in M^{5}$ there pass at most 4 distinct holomorphic curves.

Proof. We already know that there are at most 4 null lines of the Levi form in $H_{x}$ which can be tangent to a holomorphic curve. Any holomorphic curve in $M^{5}$ is the projection under $\pi: \& \rightarrow M$ of an integral of the 40 -prolongation $\varrho=\{\omega, \kappa, \bar{\kappa}, \tau\} \supseteq \mathcal{f}$. Since $\mathcal{L}$ is of corank 2 , there is at most one leaf (integral) of $\mathcal{L}$ passing through each $\xi \in \&$. Since $\&_{x} \cap \&^{\natural}$ consists of at most four points, we are done.

In the case where the four roots of $K_{x}(\theta)$ are distinct, there will generally be no holomorphic curves in $M^{5}$ anyway because further compatibility conditions must be satisfied. An interesting special case is when $K_{x}(\theta)$ has a double root (for every $x \in M$ ) which varies smoothly with $x$. In this case, it is not hard to see that the integral elements $\xi_{x} \in \&_{x}$ which correspond to the double root form an integrable distribution on $M_{5}$ and $M_{5}$ is foliated by a 3 parameter family of holomorphic curves. The CR-manifolds $M^{5}$ whose $S$-tensor satisfies this "double root" condition form an interesting special class of Lorentzian CR-manifolds. The CR-manifolds $M^{5}$ of [Penrose] are Lorentzian and seem to satisfy the "double root condition".

The "algebraically special" cases where $K_{x}(\theta)$ satisfies some multiple root hypothesis uniformly for all $x \in M$ will be discussed in a later paper.

5. Some examples. We have already remarked how the general discussion of systems of type $(m, k)$ may be applied to the problem of finding the holomorphic curves in a Lorentzian CR-manifold $M^{2 n+1}$. Explicitly, we let $X_{0}=\&$ and let $\mathscr{G}=\mathscr{g}$ and $\mathscr{K}=\mathscr{G}$ (see $\S 2$ ). Then the holomorphic curves of $M^{2 n+1}$ are represented by the

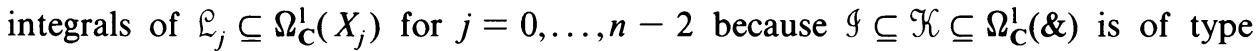
$(1, n-1)$.

In this section we give an example for each $j$ between 0 and $n-2$ of a CR-manifold $M_{j}^{2 n+1}$ such that $\varrho_{j} \subseteq \Omega_{\mathrm{C}}^{1}\left(X_{j}\right)$ is completely integrable and thus, for $j=n-2$, the holomorphic curves depend on $n^{2}$-parameters. Moreover, we will give an explicit construction of the curves in terms of Lie groups $S U(k)$.

First, we recall some standard facts about hermitian geometry and Kähler geometry.

We let $\mathbf{P}^{m}$ be projective $m$-space. The elements of $\mathbf{P}^{m}$ are complex lines in $\mathbf{C}^{m+1}$. $S U(m+1)$ acts naturally on $\mathbf{C}^{m+1}$ and therefore, by passage to the quotient it acts on $\mathbf{P}^{m}$. If we fix a line $\lambda \in \mathbf{P}^{m}$, we can represent $\mathbf{P}^{m}$ as the quotient of $S U(m+1)$ by the isotropy group of $\lambda$. We denote this projection by $\pi_{\lambda}^{m}: S U(m+1) \rightarrow \mathbf{P}^{m}$. $S U(m+1)$ preserves a Kähler form $\hat{H}_{m}$ on $\mathbf{P}^{m}$ where we choose the multiple of $\hat{H}_{m}$ which makes it a positively oriented generator of $H^{2}\left(\mathbf{P}^{n}, \mathbf{Z}\right)$. A linear inclusion $L$ : $\mathbf{P}^{k} \rightarrow \mathbf{P}^{m}$ then satisfies $L^{*} \hat{H}_{m}=\hat{H}_{k}$.

Consider now the problem of finding (even locally) a map $\phi: \mathbf{P}^{1} \rightarrow \mathbf{P}^{m}$ which is a complex map and which satisfies $\phi^{*} \hat{H}_{m}=\alpha \hat{H}_{1}$ for some given constant $\alpha$. It is an 
easy consequence of the Second Main Theorem (see [Griffiths]) that such a mapping exists if and only if $\alpha$ is an integer between 0 and $m$. In fact, it is easy to see that if $0 \leqslant \alpha \leqslant m$ and $\alpha$ is an integer then $\phi\left(\mathbf{P}^{1}\right) \subseteq \mathbf{P}^{\alpha}$ for some unique $\mathbf{P}^{\alpha} \subseteq \mathbf{P}^{m}$.

We can construct the solutions to this problem explicitly via representation theory. Let $\psi_{\alpha}: S U(2) \rightarrow S U(\alpha+1)$ be the unique irreducible representation of $S U(2)$ of degree $(\alpha+1)$. We can specify $\psi_{\alpha}$ uniquely by describing the image of the maximal torus $U(1) \subseteq S U(2)$.

In fact, we require

$$
\psi_{\alpha}\left(\begin{array}{ll}
e^{i \theta} & 0 \\
0 & e^{-i \theta}
\end{array}\right)=\left(\begin{array}{lll}
e^{i \alpha \theta} & & 0 \\
& \ddots & \\
0 & & e^{-i \alpha \theta}
\end{array}\right) .
$$

Choosing arbitrary $\eta \in S U(\alpha+1), \lambda_{1}=(1,0)$ in $\mathbf{C}^{2}$ and $\lambda_{\alpha}=(1,0, \ldots, 0)$ in $C^{\alpha+1}$, the map $\tilde{\gamma}_{\eta}^{\alpha}: S U(2) \rightarrow S U(\alpha+1)$ given by $\tilde{\gamma}_{\eta}^{\alpha}(g)=\eta \psi^{\alpha}(g)$ descends via the maps $\pi_{\lambda_{1}}^{1}: S U(2) \rightarrow \mathbf{P}^{1}$ and $\pi_{\lambda_{\alpha}}^{\alpha}: S U(\alpha+1) \rightarrow \mathbf{P}^{\alpha+1}$ to a map $\gamma_{\eta}^{\alpha}: \mathbf{P}^{1} \rightarrow \mathbf{P}^{\alpha}$ which can be shown to satisfy $\gamma_{\eta}^{\alpha *} \hat{H}_{\alpha}=\alpha \hat{H}_{1}$. For each $[\eta] \in S U(\alpha+1) / Z_{\alpha+1}$, we get a different curve so it follows that there is an $(\alpha+1)^{2}-1=\alpha^{2}+2 \alpha$ (real) parameter family of solutions to the problem in $\mathbf{P}^{\alpha}$. Since there is a $2(m-\alpha)(\alpha+1)$ (real) parameter family of $\mathbf{P}^{\alpha} \subseteq \mathbf{P}^{m}$, it follows that the original problem of finding the complex maps $\phi: \mathbf{P}^{1} \rightarrow \mathbf{P}^{m}$ with $\phi^{*} \hat{H}_{m}=\alpha \hat{H}_{1}$ has a grand total of

$$
\alpha^{2}+2 \alpha+2(m-\alpha)(\alpha+1)=2 m(\alpha+1)-\alpha^{2}
$$

(real) parameter family of solutions.

With this in mind, we let $n \geqslant 2$ be fixed. For each $p, q$ positive integers, we let $\Omega_{p, q}=q \hat{H}_{n-1}-p \hat{H}_{1}$ be defined as a (pseudo) Kähler-form on $\mathbf{P}^{1} \times \mathbf{P}^{n-1}$. Because $\Omega_{p, q}$ is integral and of type $(1,1)$, there exists a holomorphic line bundle $L_{p, q} \rightarrow \mathbf{P}^{1} \times$ $\mathbf{P}^{n-1}$ whose Chern class is exactly $\Omega_{p, q}$. Let an hermitian structure be fixed on $L_{p, q}$ and let $M_{p, q}^{2 n+1} \subseteq L_{p, q}$ be the circle bundle of unit vectors in $L_{p, q}$. Finally, let $\alpha \in$ $\Omega^{1}\left(M_{p, q}^{2 n+1}\right)$ be the admissible connection form of $L_{p, q}$. By definition, $\alpha$ is a real 1 -form which restricts to $d \theta / 2 \pi$ on the fibers of the map $\pi_{p, q}: M_{p, q}^{2 n+1} \rightarrow \mathbf{P}^{1} \times \mathbf{P}^{n-1}$ which satisfies $d \alpha=i \Omega_{p, q}$.

Let $I_{p, q}$ be the complex Pfaffian system on $M_{p, q}^{2 n+1}$ generated by $\alpha$ and the pullback under $\pi_{p, q}$ of the $(1,0)$ forms on $\mathbf{P}^{1} \times \mathbf{P}^{n-1}$. It is clear from the above formula that $\left(M_{p, q}^{2 n+1}, I_{p, q}\right)$ is a CR-manifold of Lorentzian type. Moreover, we can describe the holomorphic curves in $M_{p, q}^{2 n+1}$ explicitly. Suppose that $\phi: N \rightarrow M$ is a holomorphic curve in $M_{p, q}^{2 n+1}$. Then it is a consequence of our definitions that $\pi_{p, q} \circ \phi: N \rightarrow \mathbf{P}^{1} \times \mathbf{P}^{n-1}$ is a complex curve which is an integral of $\Omega_{p, q}$. Now $\phi^{*} \circ \pi_{p, q}^{*}\left(\hat{H}_{1}\right) \neq 0$ because otherwise $\pi_{p, q} \circ \phi$ would necessarily have rank 0 , which is a contradiction. It follows that we may locally regard $\pi_{p, q} \circ \phi(N) \subseteq \mathbf{P}^{1} \times \mathbf{P}^{n-1}$ as the graph of a holomorphic map $\psi: \mathbf{P}^{1} \rightarrow \mathbf{P}^{n-1}$. The condition that $\left(\pi_{p, q} \circ \phi\right)^{*}\left(\Omega_{p, q}\right)$ $=0$ translates into the condition $\psi^{*}\left(\hat{H}_{n-1}\right)=(p / q) \hat{H}_{1}$.

This immediately gives

Proposition 5.1. $M_{p, q}^{2 n+1}$ has no holomorphic curves at all unless $p / q$ is an integer between 1 and $n-1$. 
Conversely, let us now assume that $p / q=k$ where $k$ is an integer between 1 and $n-1$. Then the complex integrals of $\Omega_{p, q}$ in $\mathbf{P}^{1} \times \mathbf{P}^{n-1}$ are just the graphs of maps $\psi: \mathbf{P}^{1} \times \mathbf{P}^{n-1}$ which satisfy $\psi^{*} \hat{H}_{n-1}=k \hat{H}_{1}$. We know from earlier discussions that these maps depend on $2(n-1)(k+1)-k^{2}$ real parameters. Given an integral $\phi$ : $N \rightarrow \mathbf{P}^{1} \times \mathbf{P}^{n-1}$ of the form $\Omega_{p, q}$, there is a one parameter family of lifts $\tilde{\phi}(\theta)$ : $N \rightarrow M_{p, q}^{2 n+1}$ which satisfy $(\tilde{\phi}(\theta))^{*}(\alpha)=0$. The reason is that the connection $\alpha$ is flat over $\phi(N) \subseteq \mathbf{P}^{1} \times \mathbf{P}^{n-1}$ by hypothesis. Therefore

Proposition 5.2. If $p / q=k$ is an integer between 1 and $n-1$, then $M_{p, q}^{2 n+1}$ has a $2(n-1)(k+1)+1-k^{2}$ parameter family of holomorphic curves.

REMARKs. The parameter space for the holomorphic curves is connected, and, in fact, is easily seen to be a quotient space of $S U(n)$.

If $k=n-1$, then the number of parameters is exactly $n^{2}$, the maximum predicted by our theory in $\S 3$.

If $p / q=k$ (an integer between 1 and $n-1)$, it is not difficult to show that the system $\varrho_{k-1}$ in $\Omega_{\mathbf{C}}^{1}\left(X_{k-1}\right)$ is completely integrable.

Returning to the general case, it is not difficult to show, using Chern-Moser theory, that if $\varrho_{0}$ is completely integrable on $X_{0}=\&(M)$, then $M$ must be locally equivalent to the hyperquadric (Example 1.5) in $\mathbf{C}^{n+1}$. This raises the interesting question of whether the complete integrability of $\mathcal{L}_{j_{0}} \subseteq \Omega_{\mathbf{C}}^{1}\left(X_{j_{0}}\right)$ (we assume $X_{j_{0}} \neq \varnothing$ ) where $X_{0}=\&(M)$ implies that $M$ is locally equivalent to $M_{j_{0}, 1}^{2 n+1}$. More generally, one might ask if the complete integrability of $\mathscr{L}_{j_{0}} \subseteq \Omega_{\mathbf{C}}^{1}\left(X_{j_{0}}\right)$ implies some standard normal form for an arbitrary system of type $(m, k)$ (not just for those systems arising from CR-manifolds). These questions appear to be very subtle. When $j_{0}=1$, our proof for CR-manifolds requires the Chern-Moser theory. In the case $j_{0}=2$, even the Chern-Moser theory does not seem to be helpful; when one expresses the integrability of $\mathscr{L}_{2}$ in terms of Chern-Moser invariants, one arrives at equations which are quadratic and cubic in the $S$-tensor and its first two covariant derivatives. Straightforward analysis of these equations leads to enormous algebraic problems.

A more reasonable approach seems to be to use the integrals of $\varrho_{j_{0}}$ to define a path geometry in $M$ in the sense of Engel and [Cartan]. One then uses these paths to define a mapping from $M$ to $M_{j_{0}, 1}^{2 n+1}$ by a higher order cross-ratio formula. This method seems to be more manageable, but the picture is far from complete.

\section{REFERENCES}

R. Bryant, S. S. Chern and P. A. Griffiths, Notes on exterior differential systems, Proc. 1980 Peking Symposium on Differential Equations, Peking University, 1980.

E. Calabi, Minimal immersions of surfaces in Euclidean spheres, J. Differential Geom. 1 (1967), 111-127.

E. Cartan, "Le calcul de variations et certaines familles de courbes" in Oeuvres, Vol. II, Gauthier-Villars, Paris, 1983, pp. 1011-1034.

S. S. Chern, Complex manifolds without potential theory, 2nd ed., Springer-Verlag, Berlin and New York, 1979.

S. S. Chern and J. Moser, Real hypersurfaces in complex manifolds, Acta Math. 133 (1974), 219-271. 
P. A. Griffiths, On Cartan's method of Lie groups and moving frames as applied to existence and uniqueness questions in differential geometry, Duke Math. J. 41 (1974), 775-814.

H. B. Lawson, Jr., Minimal varieties in constant curvature manifolds, Ph.D. Thesis, Stanford, Calif., 1968.

L. Nirenberg, On a question of Hans Lewy, Uspehi Mat. Nauk 29 (1965), 251-262.

R. Penrose, Twistor algebra, J. Math. Phys. 8 (1967), 345-366.

F. Sommer, Komplex-analytische Blätterung reeller Hyperflächen im $\mathbf{C}^{n}$, Math. Ann. 137 (1959), S $392-411$.

Department of Mathematics, Rice University, Houston, TeXas 77001 